CORPUS $\begin{aligned} & \text { Corpus } \\ & 21 \mid 2020 \\ & \text { Dispositifs numériques et dévoilement de soi }\end{aligned}$

\title{
Ces mots que Macron emprunte à Sarkozy. Discours et intelligence artificielle
}

These words that Macron borrows from Sarkozy. Discourse and Artificial Intelligence

Damon Mayaffre, Magali Guaresi et Laurent Vanni

C OpenEdition

Édition électronique

URL : http://journals.openedition.org/corpus/5105

DOI : $10.4000 /$ corpus. 5105

ISSN : 1765-3126

Éditeur

Bases; corpus et langage - UMR 6039

Référence électronique

Damon Mayaffre, Magali Guaresi et Laurent Vanni, « Ces mots que Macron emprunte à Sarkozy.

Discours et intelligence artificielle», Corpus [En ligne], 21 | 2020, mis en ligne le 09 mars 2020,

consulté le 25 janvier 2021. URL : http://journals.openedition.org/corpus/5105 ; DOI : https://doi.org/

$10.4000 /$ corpus. 5105

Ce document a été généré automatiquement le 25 janvier 2021.

(c) Tous droits réservés 


\section{Ces mots que Macron emprunte à Sarkozy. Discours et intelligence artificielle}

These words that Macron borrows from Sarkozy. Discourse and Artificial

Intelligence

Damon Mayaffre, Magali Guaresi et Laurent Vanni

\section{Introduction}

Décrypter le discours de Macron à l'Elysée passe par une approche comparative et en l'occurrence généalogique. C'est dans la comparaison historique systématique et raisonnée avec de Gaulle, Pompidou, Giscard, Mitterrand, Chirac, Sarkozy et Hollande que se trouve la clef du mystère verbal que constitue le nouveau président. Discours de gauche ou discours de droite? Discours vertical ou discours horizontal? Discours jacobin ou discours girondin?

2 Lors de son investiture à l'Elysée, le 14 mai 2017, il ne manque pas de mentionner ses 8 devanciers avant d'endosser solennellement son costume :

Je songe au Général de Gaulle, qui œuvra pour redresser la France et lui rendre son rang dans le concert des nations. Je songe à Georges Pompidou, qui fit de notre pays une puissance industrielle majeure. À Valéry Giscard d'Estaing, qui sut faire entrer la France et sa société dans la modernité. À François Mitterrand, qui accompagne la réconciliation du rêve français et du rêve européen. À Jacques Chirac, nous donnant le rang d'une nation sachant dire non aux prétentions des va-t-en-guerre. À Nicolas Sarkozy, ne comptant pas son énergie pour résoudre la crise financière qui avait si violemment frappé le monde. Et je songe bien sûr à François Hollande, faisant œuvre de précurseur avec l'Accord de Paris sur le climat et protégeant les Français dans un monde frappé par le terrorisme. ${ }^{1}$

3 L'hommage aux Pairs paraitra peut-être convenu puisque Nicolas Sarkozy en 2007 et Français Hollande en 2012 l'avait déjà prononcé presque mot pour mot, 5 ans et 10 ans auparavant ${ }^{2}$, mais une hypothèse forte voit le jour : loin d'être une nouveauté, Macron 
est un héritage. Moins l'homme d'une thèse originale et inédite, Macron est une synthèse qui s'applique à tenir un discours qui emprunte ses mots à tous ses devanciers, consciemment souvent, inconsciemment parfois, par élan naturel ou mimétisme revendiqué.

Et dans ce cadre, c'est Nicolas Sarkozy qui est quantitativement son préteur privilégié à la fois d'un point de vue thématique (la célébration du travail qu'il convient d'augmenter, de sa valeur émancipatrice, de sa nécessaire désindexation face aux cotisations et à la fiscalité) que d'un point de vue stylistique ou énonciatif (une égoprésidence, volontaire sinon autoritaire, jupitérienne sinon martiale). C'est donc l'empreinte-Sarkozy sur le discours d'Emmanuel Macron que cet article établit et décrit.

\section{Méthodologie}

5 En 30000 signes, cette contribution entend être uniquement analytique sur le discours présidentiel d'Emmanuel Macron : elle relève de l'analyse du discours politique.

6 Pour les présupposés méthodologiques de la Logométrie et du Deep learning qui seront utilisés, ainsi que sur les présupposés conceptuels sur la notion d'intertexte, de corpus réflexifs, de texte-palimpseste, de parcours interprétatif ou d'herméneutique numérique, nous ne pouvons que renvoyer le lecteur à la bibliographie et, en ce qui nous concerne, à nos publications les plus récentes (2018-2020) sur ces questions : Guaresi 2020, Guaresi et al. 2020-sous presse, Mayaffre et al. 2019, Mayaffre 2020, Mayaffre et al. 2020a, Mayaffre et al. 2020b, Vanni et al. 2018a, Vanni et al. 2018b, Brunet et Vanni 2019, Vanni et al. 2020 et Vanni et al. 2020-submitted.

7 Admettons simplement ici que l'Intelligence artificielle des ordinateurs et la Logométrie permettent de détecter automatiquement dans le corpus présidentiel (1958-2020), les emprunts linguistiques et les originalités discursives des présidents, les ressemblances et les différences. Sur un corpus de presque 1100 discours et de 4 millions de mots, la machine a ainsi appris à reconnaitre le de Gaulle (des phrases longues autour de 35 mots, de nature nominale ou adjectivale, avec notamment la «France", le "peuple», "l'Etat» comme premiers noms) comme elle a appris à reconnaitre le Mitterrand (phrases courtes autour de 25 mots, de nature verbale et pronominale, avec le «moi» et le «je» comme centre d'intérêt personnel et «l'Europe » comme horizon). Elle a appris à reconnaitre le Sarkozy (un lexique fort et une syntaxe faible) et le Hollande (une syntaxe compliquée et un lexique affadi). Elle a appris à reconnaitre le Giscard (discours technocratique ou énarchique) ou le Pompidou (discours littéraire, riche voire ampoulé). Et il est dès lors facile de demander à Hyperbase ${ }^{3}$ ce que Macron emprunte aux uns et aux autres, ou comment, au contraire, il se démarque de chacun.

8 Le deep learning appliqué au texte peut ainsi chiffrer ces emprunts multiples : après apprentissage, l'algorithme évalue chacun des 10000 paragraphes du corpus Macron (2017-2020) pour leur trouver une forme de paternité ou source d'inspiration dans le corpus présidentiel (1958-2017). Ainsi, supposons que Macron prononce une phrase comme « Notre planète est en feu et nous regardons ailleurs » ou «La France brule et nous regardons à côté ». Alors le logiciel identifiera automatiquement, après examen $\mathrm{du}$ lexique, de la grammaire et de la syntaxe, une ressemblance linguistique avec 
Jacques Chirac lors de son fameux discours à Johannesburg en 2002. Supposons maintenant que Macron s'écrie à la tribune: "Je vous ai entendus!». Hyperbase détectera l'inspiration gaulliste d'un discours de juin 1958 à Alger ; etc.

Sur les 10000 phrases du corpus Macron, les taux de reconnaissance se hiérarchisent ainsi :

Tableau 1. Sources d'inspiration en \% de phrases du discours de Macron (Hyperbase)

\begin{tabular}{|l|l|l|}
\hline & Présidents inspirateurs & Taux d'inspiration \\
\cline { 1 - 3 } & De Gaulle & $2 \%$ \\
\cline { 2 - 3 } Discours d'Emmanuel Macron & Pompidou & $7 \%$ \\
\cline { 2 - 3 } & Giscard & $3 \%$ \\
\cline { 2 - 3 } & Mitterrand & $12 \%$ \\
\cline { 2 - 3 } & Chirac & $21 \%$ \\
\cline { 2 - 3 } & Sarkozy & $29 \%$ \\
\cline { 2 - 3 } & Hollande & $26 \%$ \\
\hline
\end{tabular}

10 Les taux d'emprunts ou d'inspiration répondent ainsi grosso modo à une logique temporelle ou chronologique dans le corpus. En règle générale, le discours de Macron ressemble d'autant plus à celui d'un de ses prédécesseurs que celui-ci a présidé récemment et d'autant moins que celui-ci a présidé il y a longtemps : aux deux opposés par exemple, Macron s'inspire seulement à hauteur de $2 \%$ de De Gaulle alors qu'il s'inspire à hauteur de $26 \%$ de Hollande.

11 Pourtant, d'intéressantes exceptions contredisent cet agencement chronologique : d'une part Pompidou inspire plus Macron que Giscard, nous ne pourrons y revenir ici (cf. Mayaffre 2020). Surtout - et c'est l'objet de cette contribution - Sarkozy, en tête du classement avec $29 \%$, inspire plus le nouveau président que quiconque, et que Hollande pourtant son immédiat prédécesseur, avec qui il a travaillé étroitement.

12 Au-delà du classement et de la première place remarquable accordée à Sarkozy, il devient intéressant d'analyser les phrases et unités linguistiques que le réseau de neurones artificiels repère comme communes au président de droite et au président du centre ${ }^{4}$.

\section{Un nouveau président des riches ? Sarkozy et le «travaillez plus »}

Dans la chronologie du quinquennat Macron, les premières mesures économiques et fiscales ont essuyé un tir de barrage de la gauche. Et l'angle d'attaque a été presque toujours le même: à 10 ans de distance, Macron imiterait Sarkozy pour mener une politique libérale favorable aux grandes entreprises et aux plus fortunés. 
Je veux ensuite miser sur le travail. Le travail est au cœur de notre société d'abord parce qu'il est ce qui permet à chacun de trouver sa place, de progresser dans la vie, de s'émanciper de son milieu d'origine si c'est la volonté que chacun porte, mais c'est aussi par le travail que notre Nation sera plus forte parce qu'elle produira, parce qu'elle s'enrichira; nous avons besoin du travail et je le défendrai sans relâche en permettant à chaque travailleur de gagner davantage par celui-ci, en formant nos concitoyens qui sont au chômage pour qu'ils puissent retrouver un travail et les compétences nécessaires pour cela, en formant nos jeunes par l'apprentissage ; le travail est le cœur de notre projet en commun. ${ }^{10}$

Ce passage, la machine se laisse abuser, n'appartient pas à Nicolas Sarkozy mais bien à Emmanuel Macron lors de ses premiers vœux aux Français le 31 décembre 2017. Et de cet hymne au travail - le mot est prononcé six fois en quelques lignes - nous retiendrons moins le "gagner davantage » (Macron) déjà entendu sous la forme du "gagner plus» (Sarkozy), que le verbe fort "s'émanciper», que nous retrouvons souvent, chez les deux présidents, dans la forme nominale : « émancipation ». 
20 À un moment où Macron se trouve contesté par un mouvement social inédit sous la Ve République, qui semble ébranler le système tout entier, le président prend de manière solennelle la parole à la télévision le 10 décembre 2018, et semble alors se réfugier dans ces dernières certitudes :

C'est d'abord l'état d'urgence économique et sociale que je veux décréter aujourd'hui. Nous voulons bâtir une France du mérite, du travail, une France où nos enfants vivront mieux que nous. Cela ne peut se faire que par une meilleure école, des universités, de l'apprentissage et des formations qui apprennent aux plus jeunes et aux moins jeunes ce qu'il faut pour vivre libre et travailler. ${ }^{11}$

21 Or pour Sarkozy, le travail, on s'en rappelle, est un moyen de s'enrichir bien sûr («travailler plus pour gagner plus», il faudra y revenir), mais plus hautement une question morale ou éthique, une question d'épanouissement, une question de « valeur $»^{12}$ :

Bien sûr qu'en 2008, nous poursuivrons la politique de revalorisation du travail, parce que le travail, c'est une valeur essentielle, c'est ce par quoi chacun trouve sa place dans la société, c'est ce qui tisse du lien social, le travail, ce n'est pas un asservissement, le travail, c'est une émancipation. Le travail, c'est un moyen de conquérir sa liberté. ${ }^{13}$

"Vivre libre et travailler» (Macron), « le travail, c'est un moyen de conquérir sa liberté » (Sarkozy), « s'émanciper » (Macron), « émancipation » (Sarkozy) : les deux présidents partagent, hautement, une vision positive et hégélienne du travail, de la peine, du mérite, de l'effort qui possèdent selon les deux présidents, loin de l'aliénation dénoncée dans la pensée marxiste, une vertu libératoire et émancipatrice. Macron le dit dans les exacts termes de Sarkozy devant le Parlement réuni à Versailles :

L'émancipation passe aussi par le mérite et par le travail. ${ }^{14}$

En réalité, cette thématique de la libération de l'individu par le travail rapproche Macron et Sarkozy dès leur campagne électorale respective en 2017 et 2007.

En 2017, Macron résume son projet en ces mots :

Notre projet, c'est un projet de liberté par le travail et dans le travail. C'est ainsi que la vraie liberté, que l'opportunité se reconstruit. Le cœur de notre projet, c'est le travail. $^{15}$

Ou de manière plus développée :

Alors, parce que je veux libérer, je veux d'abord libérer la capacité d'entreprendre, de faire. Ce sont toutes les simplifications en termes de création d'entreprise, que nous continuerons à égrener. C'est aussi les simplifications drastiques que nous mènerons pour les entrepreneurs du quotidien que sont les indépendants. Parce que le premier entrepreneur, c'est un indépendant. [...] Être salarié, c'est accepter une relation de subordination en échange d'une garantie d'emploi. Être indépendant, entrepreneur, profession libérale, c'est avoir décidé d'être LIBRE en contrepartie d'un risque, qui est celui de ne rien avoir le lendemain [...]. Parce que je crois justement dans la liberté par le travail et l'entrepreneuriat, je l'ai dit, je veux simplifier notre système, donc je supprimerai le crédit d'impôt compétitivitéemploi pour le transformer en allègement de charges pour tout le monde. [...] libérer par le travail, c'est ça, ce qu'on veut faire. Cela veut dire que nous croyons dans une valeur fondamentale, en effet: le travail [...]. Ce que je veux construire et qui est cet esprit de conquête, c'est la liberté par le travail, et donc une capacité à libérer dans l'entreprise [...]. Pour libérer, émanciper par le travail, je veux que nous simplifiions drastiquement notre droit du travail. ${ }^{16}$

Là où Sarkozy en 2007 avait exalté le travail avec enthousiasme et prononcé des extraits assez proches : 
La politique de la vie, c'est celle qui permet à tous les hommes de travailler et de s'émanciper par le travail. Je veux rendre au travail sa valeur morale et sa capacité d'émancipation. ${ }^{17}$ "récompense » que Macron ressuscite, Sarkozy n'avait eu de cesse d'en vanter les propriétés lorsqu'il dominait la scène politique : il s'agit sans doute de la meilleure définition du sarkozysme ou désormais du sarko-macronisme. Du reste, dans le détail, pour que l'inspiration soit complète, Macron reprend parfois le ton polémique de l'ancien président de droite en n'hésitant pas à culpabiliser les Français, comme en plein malaise des Gilets jaunes, le 11 janvier 2019, dont il donne une interprétation hardie au regard de la conjoncture tendue qu'il s'agissait pourtant d'apaiser :

Le défi est que notre jeunesse trouve toute sa place dans et par le travail. Et qu'elle trouve toute sa place par l'engagement, le sens de l'effort... et le sens de cet engagement qui fait qu'on n'a rien dans la vie s'il n'y a pas cet effort. Les troubles que notre société traverse sont parfois dûs et liés au fait que beaucoup trop de nos concitoyens pensent que l'on peut obtenir sans que cet effort soit apporté [...]. Et s'il n'y a pas cet engagement, ce sens de l'effort, le fait que chaque citoyen apporte sa pierre à l'édifice par son travail, par son engagement au travail notre pays ne pourra jamais recouvrer sa force, sa cohésion... ${ }^{18}$

Concrètement, lorsqu'il s'agit de traduire cette philosophie du travail en discours simples et en actes législatifs effectifs, les phrases de Sarkozy et de Macron se confondent à s'y méprendre :

Sarkozy :

Je suis bien content d'être dans cette ville, dans ce département, dans cette région où les gens d'ici connaissent les valeurs du travail, du mérite, de l'effort. ${ }^{19}$ Macron :

L'objectif c'est le mérite, le travail. C'est ça la clé et que le travail paye mieux dans notre pays. ${ }^{20}$

Dans une de ses premières mesures fiscales contestées, Emmanuel Macron augmente la CSG sur les retraités en précisant : "Leur effort permet de récompenser le travail »" L'objectif était alors selon le président de transférer une partie des charges des salariés ou des actifs vers les pensionnés ou retraités. Or Sarkozy, dans une prose moins habile puisqu'il encourage sans ambages les retraités à reprendre un travail et assimile un instant la retraite à une « assistance » - disait plus crûment :

Et c'est la raison pour laquelle je souhaite permettre aux Français de cumuler la retraite et un emploi parce que l'on a besoin de davantage de travail en France, pas de moins de travail, parce qu'on a besoin de davantage de mérite et de récompense, pas de plus d'assistance. ${ }^{22}$

Partant de ce discours commun sur le travail que l'ordinateur a bien repéré, toutes les mesures se trouvent justifiées : la suppression de l'impôt de Solidarité sur la Fortune (ISF) ou sa transformation en Impôt sur la Fortune Immobilière (IFI), l'érection d'un bouclier fiscal, l'exonération sociale des heures supplémentaires, la flat taxe, la prime d'activité, les aides aux entreprises, le CICE et sa transformation, la baisse des charges 
patronales. Par exemple, pour annoncer ce que ses opposants de gauche qualifient de légalisation du travail au noir, Macron précise :

Je veux renouer avec une idée juste : que le surcroît de travail accepté constitue un surcroît de revenu; les heures supplémentaires seront versées sans impôts ni charges dès 2019. ${ }^{23}$

$\mathrm{Ou}$

Il faudra travailler d'avantage... Il faudra travailler plus... Pour encourager à travailler d'avantage, on a défiscalisé, désocialisé pour les travailleurs les heures supplémentaires. ${ }^{24}$

Et Sarkozy avait dit, toujours dans un style plus direct

Que les gens souhaitent faire 35 heures s'ils le veulent, tant mieux. Mais si vous voulez travailler plus pour gagner plus et faire des heures supplémentaires, pourquoi vous en empêcher? C'est quand même une idée invraisemblable. Et maintenant sur la feuille de paie de ceux qui font des heures supplémentaires... il n'y a pas de soustraction puisqu'elles sont défiscalisées... ${ }^{25}$

Ces mesures sarko-macroniennes, notamment la baisse des cotisations patronales ou salariales sur le travail, semblent prendre le risque d'assécher les ressources de l'Etatprovidence c'est-à-dire de mettre à mal le financement du système de santé ou des caisses de retraites, mais le choix politique est fort, net et assumé par les deux présidents qui ne cachent pas leurs postulats libéraux, au nom de la valorisation nécessaire du travail sans laquelle rien n'est pour eux envisageable : « au lieu de mettre de l'argent pour que les gens travaillent moins, on met de l'argent pour que les gens travaillent plus $»^{26}$ résume Sarkozy. Il s'agit là de l'expression économique simple et concrète de la théorie du ruissellement qui préside aussi bien au quinquennat Sarkozy qu'au quinquennat Macron: un Etat sans doute moins providence et redistributeur, mais un Etat davantage facilitateur de la libre-entreprise pour une plus grande production de richesse, via le travail, qui in fine doit profiter à tous.

Et laissons le traitement quantitatif et Hyperbase conclurent. En 60 ans de $\mathrm{V}^{e}$ République, nul dans le corpus plus que Sarkozy et Macron n'ont martelé avec une telle conviction le verbe "travailler» et le substantif «travail» (fig. 1); Nicolas Sarkozy avec un peu plus de force qu'Emmanuel Macron.

Figure 1. «Travail » et « Travailler » dans le corpus présidentiel (Hyperbase)

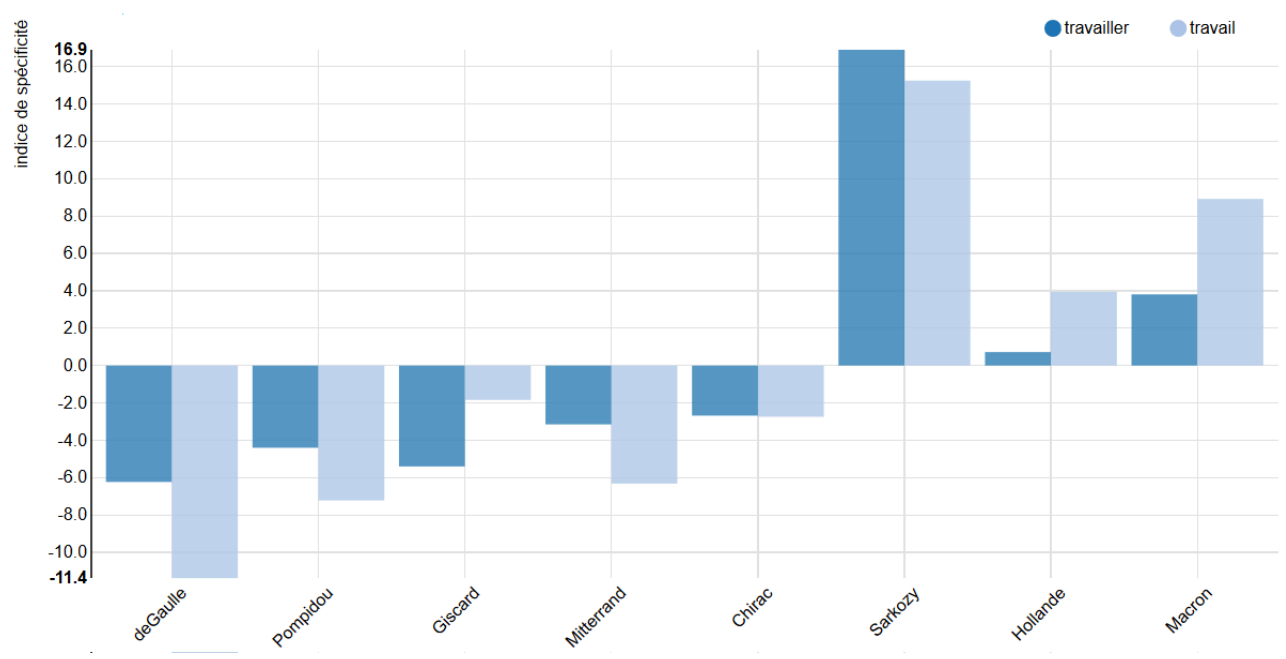


mai 2008, Sarkozy confessait à la télévision : « je suis au travail tous les jours... » et Macron reprend strictement la formule, face caméra, pour présenter ses vœux en 2018 : «Je suis au travail, fier de notre pays... ». En 2009, Sarkozy rappelait que le salaire, pour les Français, était le nécessaire "fruit de leurs efforts ${ }^{27}$, et Macron de s'inquiéter en 2019 que l'impôt prive « les travailleurs du fruit de leurs efforts $»^{28}$; ou encore, simple déclinaison, les deux croquent souvent, seuls et ensemble dans le corpus, dans « le fruit du travail $\aleph^{29}$. De manière décisive, en 2007, durant la campagne électorale, Sarkozy trouve son slogan fondateur qu'il répète une vingtaine de fois de meeting en meeting puis reprend tout le long de son quinquennat : «travailler plus pour gagner plus » ou «travailler plus pour gagner davantage ». Et Macron de réinventer l'adage quelques années plus tard, dans une formulation d'apparence différente et moins tendue, mais dans laquelle l'algorithme repère sans difficulté la même essence: «On ne peut pas travailler moins et gagner plus $»^{30}$.

\section{L'ego-président. Jupiter au-delà de Mars}

présidentialisation de la $\mathrm{V}^{\mathrm{e}}$ République - c'est-à-dire le rôle toujours plus important accordé à la personne du président dans la vie politique française - est inscrite dans ses gènes gaullistes ${ }^{31}$. Et l'histoire récente a accéléré un processus de personnalisation - ou pouvoir vertical dont le sommet se trouve à l'Elysée - que l'on observe à l'échelle mondiale dans toutes les démocraties contemporaines. Les deux principales raisons qui conspirent ensemble au phénomène ont bien été identifiées au-delà du triomphe civilisationnel de l'individualisme - ici le leader qui s'exhibe en tant que tel - qui pétri avec de plus en plus de force les sociétés modernes ${ }^{32}$.

D'un côté, la mort des alternatives politiques laisse naturellement la place à la personnalisation et aux querelles individuelles de personnes: le débat politique aujourd'hui n'est plus entre république et monarchie, démocratie et fascisme ou entre capitalisme ou communiste, mais entre personnalités qui partagent l'essentiel du modèle de référence - la démocratie libérale - et se déchirent, dès lors, autour d'ambitions personnelles. Et lorsqu'une de ces personnes accède au pouvoir, elle ne se démarque point par un programme original à l'intérieur d'un système économique globalement admis par tous mais par des traits de personnalité, une signature personnelle, une façon de faire, un ethos. Dans la technique quotidienne de nos démocraties, les partis sont ainsi devenus davantage des écuries pour candidats charismatiques que des lieux d'élaboration politique ou idéologique; dans le cas d'Emmanuel Macron notons même qu'il se lance à l'assaut du pouvoir, seul, en 2016, sans parti constitué, sans programme politique arrêté, ni corpus idéologique avoué.

De l'autre côté, l'hypermédiatisation des sociétés modernes, l'information en continue, les écrans de télévision ou de portable, les réseaux sociaux favorisent voire exigent l'incarnation. La télévision montre des corps et non des esprits, affiche les visages plus qu'elle n'expose des idées. Le président de la République en France ou les présidents des Chambres ou des Conseils dans le monde, personnifient ainsi des politiques qui au fond sont devenues secondaires dans leur contenu. Dépolitisé, sans corps intermédiaires ou rouages collectifs efficaces tel des syndicats moribonds ou des partis en perdition, le peuple s'abandonne ainsi aux stars de la politique, qu'exigent les médias, et dont la réussite personnelle semble gage d'avenir collectif. 
Dans l'arène politique française qui nous intéresse pour comprendre Emmanuel Macron, et dans le champ du discours présidentiel, ce passage à " une démocratie du public" selon les mots forts de Bernard Manin $^{33}$, dans laquelle la vedette est le président érigé en leader et les fans le peuple réduit à l'opinion publique, se fait en deux temps; Emmanuel Macron représentant ainsi le troisième temps d'un processus continu, sans doute inévitable sous le Texte de la $\mathrm{V}^{\mathrm{e}}$ République et la situation contemporaine.

Le premier temps intervient, nous ne pourrons y revenir, dans les années 1980, avec Mitterrand qui a introduit le "moi » dans le parler élyséen, en même temps qu'il enterrait l'idée ${ }^{34}$. Mitterrand a donné à sa personne une importance telle - jamais plus égalée sous la $\mathrm{V}^{\mathrm{e}}$ République (cf. graphique suivant) - qu'il se maintient au pouvoir, seul, lorsque la logique politique et les urnes l'invitaient en 1986 à démissionner. Et au terme de cette $1^{\text {re }}$ cohabitation problématique, il se représente, malade, non plus sur la foi d'un programme mais par une Lettre aux Français dans laquelle n'existait plus de proposition sinon celle de faire individuellement face à un Jacques Chirac présenté comme personnellement indigne de la fonction. Après le programme commun, substantiel et marxisant, de 1972, après les 110 propositions, précises et socialistes, de 1981, le slogan publicitaire de 1988 conçu par Jacques Séguéla, «Génération Mitterrand» disait tout de l'effondrement du contenu politique au profit d'une personnalisation, mi-infantile mi-monarchique, des enjeux.

Le deuxième temps intervient avec Sarkozy - dont Emmanuel Macron va s'inspirer dans les années 2000, pour lequel il est en même temps juste de parler de peopolisation ${ }^{35}$ de la vie politique que de présidentialisation du régime. Sans revenir aux épisodes personnels qui constituent la saga du quinquennat (divorce, remariage avec une mannequin vedette, paternité présidentielle), sans revenir non plus sur la couverture médiatique sans précédent dont il a bénéficié personnellement dans les magazines grand public ou à la télévision ${ }^{36}$, rappelons sur le fond que Sarkozy théorise, avant de la mettre à place, l'hyperprésidence c'est-à-dire la disparition ou l'effacement du $1^{\mathrm{er}}$ Ministre en faveur d'un président qui doit mener, personnellement, l'action de la majorité. Le $1^{\text {er }}$ Ministre, responsable devant le Parlement devient le simple " collaborateur $~^{37}$ d'un Président autocrate, élu au suffrage universel et sans responsabilité institutionnelle devant personne sinon lui-même et l'insaisissable opinion publique.

5 Et c'est cette ego-présidence sarkozyste plus encore que mitterrandienne que le deep learning retrouve dans les discours de Macron.

Elle se mesure avant tout par le sur-usage de la première personne du singulier qui sature les discours des trois présidents (fig. 2). 
Figure 2. « je » dans le discours présidentiel (1958-2019) (Hyperbase)

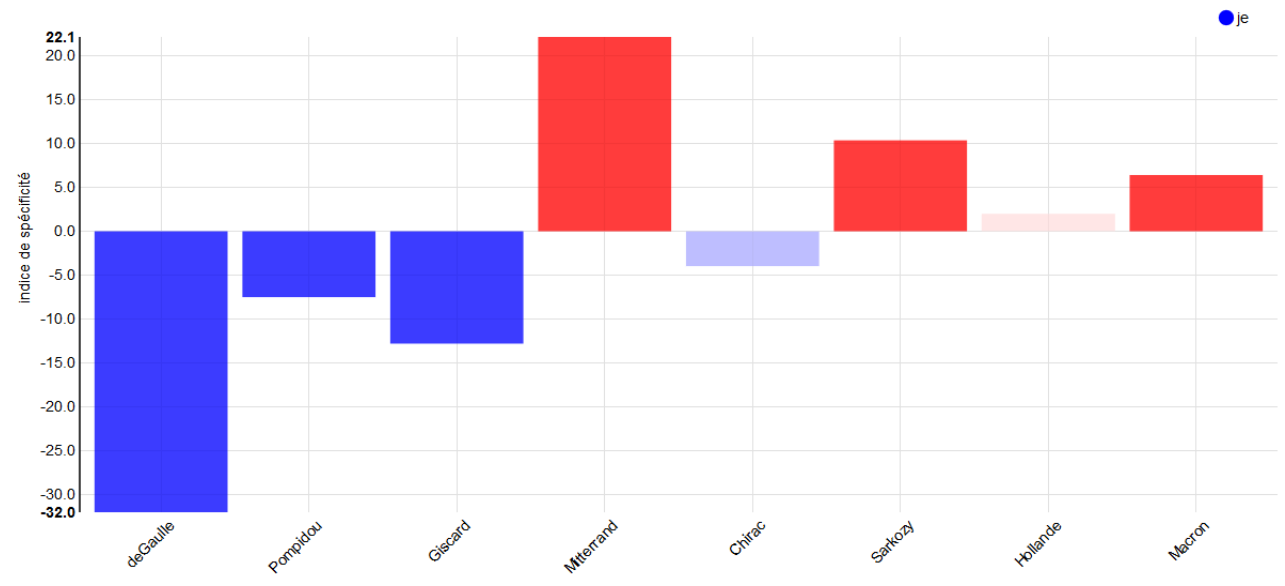

Ainsi de Gaulle disait « la France », Pompidou « la civilisation » et Giscard disait « la société ». Macron, à la suite de Mitterrand et Sarkozy - certes un peu moins qu'eux - dit «je », « moi », « mon », « ma », « mes».

Il faut regarder les verbes qui gouvernent les « je » du discours de Macron, de Sarkozy et de Mitterrand car au-delà de l'impression générale commune - une présidence personnelle -, ils apportent des nuances sur la personnalité de chacun, et rapprochent ici aussi Macron de Sarkozy.

Figure 3. Les verbes associés à « je » dans le discours présidentiel (Hyperbase)

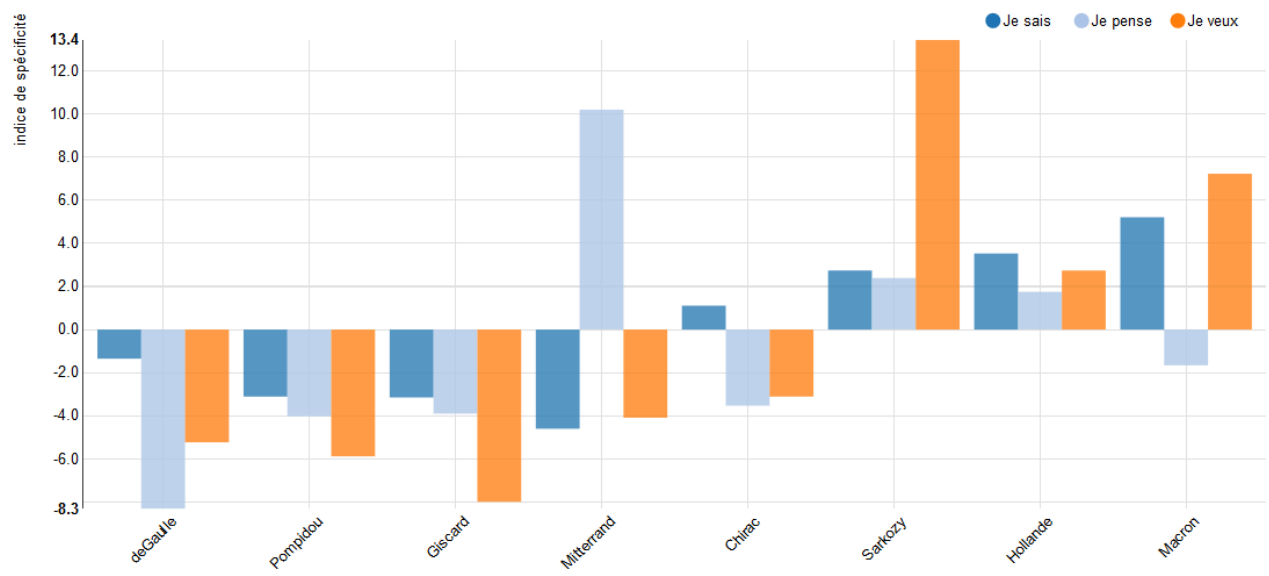

Chez Mitterrand, c'est tout entier le sujet cartésien, l'égotisme de l'intellectuel : le « je pense » domine le discours. Chez Sarkozy, c'est tout entier le sujet nietzschéen, la toute puissance du leader : le « je veux » s'exprime sans complexe. Chez Macron, c'est le sujet jupitérien, l'omniscience du guide ou du savant: le «je sais» est répété dans des proportions inégalées. Et, ce "je sais» caractéristique et original de Macron, se combine aux « je veux » sarkozystes dans une prose, au final, sans modestie.

Dans une prise de parole difficile politiquement - car adressée aux maires de France parmi lesquels peu étaient alors convertis à la République en Marche - Macron utilise cette énonciation égocentrée, celle du « je sais», celle du «je veux», celle du «moi, j'attends de vous » pour convaincre : 
Parce que je sais que vous aussi vous êtes des élus engagés et j'en ai besoin. Moi j'attends de vous que vous continuiez à entreprendre, à faire, à innover, à tester, que vous ne cédiez en rien à la morosité ambiante... ${ }^{38}$ utilisation du « je » (figure 2), du « vous » et du « nous » (figure 4)

Figure 4. « Nous » et « vous » dans le discours présidentiel (Hyperbase)

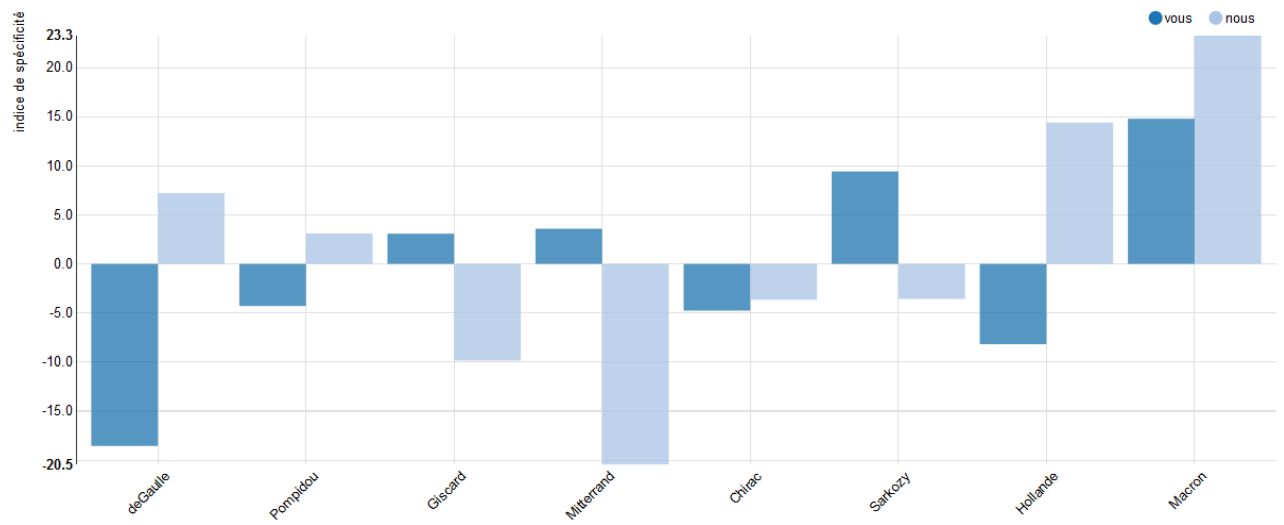

Le processus rhétorique pourra paraitre éculé puisqu'il a été souvent utilisé depuis que le discours politique existe, mais il reste de bon aloi : sans cesse Macron pose dans ses discours l'équation élémentaire « je » + « vous » = « nous ».

Nous pouvons illustrer ce parti pris rhétorique qui constitue une des clefs majeures du discours de Macron, déjà durant la campagne électorale, par exemple par la conclusion $\mathrm{du}$ discours de la victoire (nous soulignons ci-dessous les trois marques énonciatives inextricablement entremêlées de la personne (je, vous, nous)) :

Notre tâche est immense et elle imposera de construire, dès demain, une majorité vraie, une majorité forte... Cette majorité de changement, c'est cela, ce que j'attends de vous dans six semaines, car j'aurai encore et encore besoin de vous. Mes chers concitoyens, vous toutes et tous, qui êtes là, à mes côtés, depuis tant et tant de jours, tant et tant de nuits, peuple de France, ici, rassemblé au Louvre, nous avons la force, nous avons l'énergie, nous avons la volonté, celle qui nous a portés, qui a fait ce que nous sommes. C'est cela, ce qui conduira notre avenir... Je sais cette ferveur que vous portez, je sais ce que je vous dois. Et je sais ce soir ce que je dois à

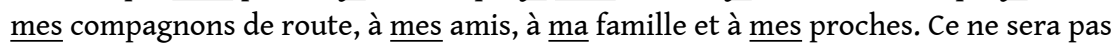
tous les jours facile, je le sais. La tâche sera dure. Le vous dirai à chaque fois la vérité. Mais votre ferveur, votre énergie, votre courage toujours me porteront. Je vous protégerai face aux menaces. ${ }^{39}$

En d'autres termes, le régime énonciatif de Macron est plus perfectionné et mieux équilibré que celui de Mitterrand et Sarkozy dominés uniquement par le « moi » ou, en sens inverse, que celui d'un Giscard dominé par l'impersonnel et l'effacement de la personne. Macron parle à la première personne comme Mitterrand et Sarkozy mais prétend parler au nom de tous dans un subtil mélange d'autorité assumée (je) et de 
représentativité (nous), c'est-à-dire d'une verticalité revendiquée (un «je» qui s'oppose à un " vous ») et d'une horizontalité souhaitée (" nous »).

Le procédé, relativement classique donc, est d'importance historique puisqu'il illustre ici, d'un point de vue discursif, le passage de la démocratie représentative à la démocratie du public décrite par Bernard Manin ${ }^{40}$, et dont Macron semble le produit le mieux achevé. Politiquement, Macron constate en effet, et accélère, la faillite des corps intermédiaires politiques et la mort de l'ancien monde démocratique fait de partis historiques et de représentants dûment constitués. De fait, l'opposition syndicale, parlementaire et partidaire à Emmanuel Macron apparait durant les deux premières années de son quinquennat comme en faillite, particulièrement les deux partis alternatifs de gouvernements (le Parti socialiste et Les Républicains) qui semblent, au moins pour le premier, en état de décomposition. De fait, Emmanuel Macron, en tout cas dans l'acte I de sa présidence jusqu'au printemps 2019, attache une importance très relative aux élus, et se fait par exemple excuser au Congrès de l'Association des Maires de France en 2018, soulevant l'indignation des communes. Dès lors, de manière quasi systématique le discours met en scène un tête à tête, sans médiation, entre le président et les Français : Macron est un président du public ; un président qui entend dialoguer directement avec le public.

La forme politique la plus remarquable de ce tête à tête, sans médiation ni partisane, ni syndicale, ni parlementaire, ni journalistique, a été la réponse que le président de la République apporte personnellement sur internet à Priscillia Ludosky, initiatrice du mouvement des Gilets Jaunes, et à travers elles aux quidams signataires de la pétition en ligne :

Chère Madame, chers tous, vous avez subi de plein fouet la hausse des prix du carburant, et avez décidé de réagir en signant cette pétition. Votre message, je l'ai entendu. Je vous réponds directement : vous avez raison... ${ }^{41}$

Et la veille d'un discours historique - celui qui devait rendre compte des conclusions du Grand débat - Macron organise un teasing laconique et remarquablement choisi sur twitter :

Demain soir, je vous réponds. ${ }^{42}$

60 Mais ce dialogue entre le « je » du président et le « vous » du public est-il efficace ? Et réussit-il réellement à construire le "nous" politique souhaité («nous la France», « nous les Européens », « nous la nation », etc.) ?

61 Malgré une élection confortable avec $65 \%$ des votants au deuxième tour qui atteste d'une capacité initiale de rassemblement, malgré un long état de grâce en début de mandat durant lequel tout semblait promis à un président sans opposition, haut dans les sondages et épargné par la presse, la chronique du quinquennat oblige à répondre par la négative, lorsqu'on se rappelle le point d'exaspération que la France a atteint durant l'automne et l'hiver 2018-2019 avec les Gilets jaunes, puis durant l'hiver 2019-2020 avec la contestation de la réforme des retraites. Le dialogue précisément semble alors rompu entre le «je» présidentiel et le gros des Français. L'incompréhension croissante, jusqu'au divorce, entre la base hexagonale et le sommet élyséen se traduit par l'effondrement de la cote de popularité du président et des slogans « Macron démission! » sont scandés lors des manifestations. À ces instants du quinquennat, Macron ne semble plus pouvoir articuler un «nous» rassembleur, large et crédible. Et dans ce moment de crise durant lequel l'unité nationale semblait en jeu, la personnalité trop forte ou égotique l'emporte dans le discours. De manière 
significative, le 10 décembre 2018, Macron prend solennellement la parole devant 22 millions de téléspectateurs et une France suspendue à ce qu'il va dire. Macron, face camera, fait alors des efforts pour paraitre modeste et à l'écoute jusqu'à formuler une forme de mea culpa. Seulement très vite dans le discours, son tempérament reprend le dessus, et les «je » et les «je veux» finissent pas l'emporter. Nous en recensons pas moins de 19 dans ce discours décisif (figure 5).

Figure 5. Quelques « je veux » du discours du 10 décembre 2018 (Hyperbase)

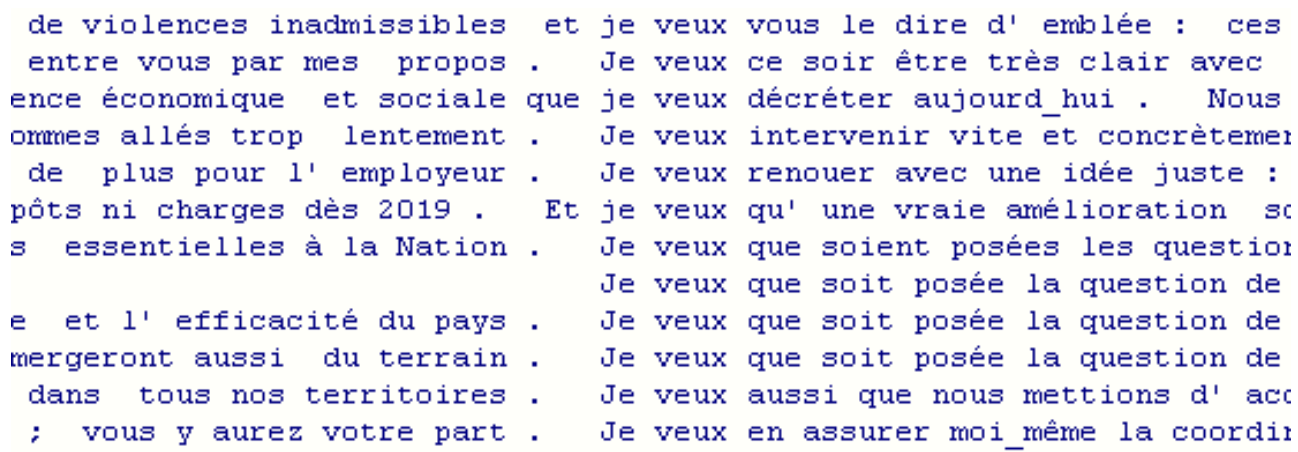

62 L'égo-présidentialité de Macron prend ainsi des tournures plus variées que celle de ces prédécesseurs, mais reste centrée sur le «moi»; et ici sur un «je veux» très sarkoziste.

63 Si elle se trouve en cohérence avec la personnalisation du pouvoir et la présidentialisation du régime en ce début de XXI ${ }^{\mathrm{e}}$ siècle, cette égo-présidentialité entre en contradiction avec l'horizontalité politique revendiquée par Macron durant la campagne 2017 de En Marche, et avec l'échange démocratique que le Président semble appeler de ses vœux notamment lors du Grand débat : la démocratie du public est une démocratie asymétrique dans laquelle par définition le Président ne saurait se contenter d'être l'égal de l'opinion publique, et où le « je » présidentiel semble toujours phagocyter le « nous » des citoyens. ${ }^{43}$

\section{Conclusion}

Après une rencontre semi-officielle entre Emmanuel Macron et Nicolas Sarkozy au printemps 2019, l'ancien président François Hollande polémique :

Je sais qu'ils se voient assez régulièrement et qu'Emmanuel Macron est aujourd'hui plus proche des idées de Nicolas Sarkozy que des miennes que ce soit socialement ou fiscalement. ${ }^{44}$

De fait dans le corpus, à tout mesurer, Emmanuel Macron s'inspire plus de Nicolas Sarkozy que de nul autre président, y compris celui dont il fut le conseiller à l'Elysée et le ministre de l'Economie.

Dans la chronologie du quinquennat cette proximité de discours apparait très rapidement - dès juillet 2017, peut-être dès la campagne électorale - autour des Ordonnances travail, des premières mesures fiscales et d'un style vertical de présidentialité dans lequel le «je veux» du président laisse peu de place au Premier ministre, au parlement, aux corps intermédiaires ou à l'opposition.

Plus tard, à cause de la situation politique ou électorale de la France, cette proximité semble se confirmer, et s'amplifier. L'opposition socialiste apparait à ce point affaiblie 
et la gauche divisée que Macron semble ne plus avoir à séduire un électorat qui saura toujours rallier la République en Marche face à la menace Front national. À l'inverse, pour préempter l'électorat d'une droite qui menace de se reconstruire ou de migrer vers l'extrême droite, le président semble s'appliquer à utiliser les mots («travail », " effort », « valeur », « récompense », etc.) et la posture de l'Autorité (« je », « je veux ») face aux Gilets Jaunes ou aux grèves contre la réforme des retraites; mots et posture que le leader historique de la droite du nouveau siècle, Nicolas Sarkozy, avait su incarner 10 années auparavant.

\section{BIBLIOGRAPHIE}

Barbet D. et Mayaffre D. (dir.) (2009). « 2007. Débats pour l'Elysée », Mots 89.

Brunet E. et Vanni L. (2019). « Deep learning et authentification des textes », Texto!, XXIV, 1

[http://www.revue-texto.net/docannexe/file/4194/texto_brunetvanni_deep_final.pdf], consulté le 06/01/2020].

Charaudeau P. (2008). Entre populisme et peopolisme. Comment Sarkozy a gagné. Paris : Vuibert, 2008.

Fairclough N. (2014 - $3^{\mathrm{e}}$ éd.). Language and Power ( $3^{\text {rd }}$ ed.). London : Longman.

Heudin J.-C. (2016). Comprendre le Deep Learning. Une introduction aux réseaux de neurones, Science eBook.

Guaresi M. (2020). « Décrire les textes politiques par le deep learning : à la recherche de nouveaux observables ", JADT2020, Toulouse.

Guaresi M. et al. (2020 - sous presse). «Intelligence artificielle et textes politiques. Quelles plusvalues interprétatives ? », in D. Mayaffre et al (dir.), L'intelligence artificielle des textes. Paris : Honoré Champion.

Jost F. et Muzet D. (2008). Le Téléprésident. La Tour-d'Aigues : L’Aube.

Lebart L., Pincemin B. et Poudat C. (2019). Analyse des données textuelles. Montréal : Presses universitaires de Québec.

Mayaffre D. (2012). Le discours présidentiel sous la Ve République. Chirac, Mitterrand, Giscard, Pompidou, de Gaulle. Paris : Presses de Sciences Po.

Mayaffre D. (2020). Macron par l'intelligence artificielle. Ses discours décryptés par la machine. La Tourd'Aigues : Les éditions de l'Aube.

Mayaffre D. et al. (2020a). « Du texte à l'intertexte. Le palimpseste Macron au révélateur de l'intelligence artificielle ", $7^{e}$ Congrès Mondial de Linguistique Française.

Mayaffre D. et al. (2020b). « Objectiver l'intertexte ? Emmanuel Macron, deep learning et statistique textuelle », JADT 2020, Toulouse.

Née E. (dir.) (2017). Méthodes et outils informatiques pour l'analyse des discours. Rennes : Presses universitaires de Rennes. 
Roussellier N. (2015). La Force de gouverner. Le pouvoir exécutif en France, XIX ${ }^{e}-X X I^{e}$ siècles. Paris : Gallimard.

Vanni L. et al. (2018a). « ADT et deep learning, regards croisés. Phrases-clefs, motifs et nouveaux observables ", in D. Iezzi et al. (dir.) JADT'2018, UniverItalia, Rome, 2018, 459-466. [hal-01823560].

Vanni L. et al. (2018b). « Text Deconvolution Saliency (TDS) : a deep tool box for linguistic analysis », 56th Annual Meeting of the Association for Computational Linguistics [hal-01804310].

Vanni L. et al. (2020). « Hyperdeep : deep learning descriptif pour l'analyse de données textuelles » ", JADT 2020, Toulouse.

Vanni L. et al. (2020-submitted). « From text saliency to linguistic objects: learning linguistic interpretable markers with a multi-channels convolutional architecture ». 57th Annual Meeting of the Association for Computational Linguistics.

\section{NOTES}

1. Macron, 14 mai 2017, discours d'investiture.

2. N. Sarkozy déclare ainsi : «Je pense au Général De Gaulle qui sauva deux fois la République, qui rendit à la France sa souveraineté et à l'Etat sa dignité et son autorité. Je pense à Georges Pompidou et à Valéry Giscard d'Estaing qui, chacun à leur manière, firent tant pour que la France entrât de plain-pied dans la modernité. Je pense à François Mitterrand, qui sut préserver les institutions et incarner l'alternance politique à un moment où elle devenait nécessaire pour que la République soit à tous les Français. Je pense à Jacques Chirac, qui pendant douze ans a œuvré pour la paix et fait rayonner dans le monde les valeurs universelles de la France » (16 mai 2007, discours d'investiture). F. Hollande en 2012 utilise également quasi les mêmes mots. A noter que Chirac en 1995 ne cite que de Gaulle et Mitterrand en oubliant Giscard et Pompidou, et que Mitterrand en 1981 cite seulement Giscard à qui il succède.

3. Hyperbase est un logiciel de Logométrie et de Deep learning. Il est produit par le CNRS et l'Université de Nice (UMR 7320, Bases, Corpus, Langage) et développé par Etienne Brunet et Laurent Vanni.

4. Les spécialistes de deep learning auront compris que le phénomène d'attribution (ou classification - tableau 1) relève d'un modèle convolutionnel lorsque la description linguistique qui va suivre des phrases relève d'un processus de déconvolution. Pour le détail scientifique et technique voir notre article: Vanni et al., Text Deconvolution Saliency (TDS) : a deep tool box for linguistic analysis. 56th Annual Meeting of the Association for Computational Linguistics, Jul 2018, Melbourne, France.

5. Michel Sapin, 25 septembre 2017, interview radio (Europe 1).

6. Cette étiquette trouve sa large diffusion par l'ouvrage à succès de Monique Pinçon-Charlot et Michel Pinçon : Le Président des riches. Enquête sur l'oligarchie dans la France de Nicolas Sarkozy, 2010, La découverte. En 2019, les sociologues rééditent leur enquête et titrent à propos de Macron : Le président des ultra-riches, Zones, 2019.

7. François Hollande, 25 avril 2018, interview télévisée (TMC).

8. Sarkozy, 27 mai 2008, interview radiophonique (RTL).

9. En anticipant l'ensemble de la démonstration rappelons que Macron déclare que sa conception du travail est pour lui une "philosophie », et précise dans la suite du discours : «Le travail n'est pas qu'un élément économique. C'est le sens que l'on donne à sa vie. » (Macron, 11 janvier 2019, discours de la Galette des rois à l'Elysée).

10. Macron, 31 décembre 2017, vœux aux Français.

11. Macron, 10 décembre 2018, allocution télévisée. 
12. Pour un développement sur la «valeur travail » chez le seul Nicolas Sarkozy voir notre étude dans un précédent ouvrage. En une sortie-machine logométrique, le premier mot statistiquement associé à « travail » en 2007 dans la bouche de Sarkozy est non pas « salaire », «travailleur » ou « condition » mais « valeur».

13. Nicolas Sarkozy, 08 janvier 2008, conférence de presse télévisée.

14. Macron, 9 septembre 2018, discours au Congrès à Versailles.

15. Macron, 13 avril 2017, meeting à Pau.

16. Macron, 28 février 2017, meeting à Angers.

17. Sarkozy, $1^{\mathrm{er}}$ mars 207 , meeting à Bordeaux.

18. Macron, 11 janvier 2018, discours de la Galette des rois à l'Elysée.

19. Sarkozy, 24 mars 2009, discours à Saint-Quentin.

20. Macron, 12 avril 2018, interview télévisée (TF1).

21. Macron, 31 août 2017, interview dans Le Point.

22. Nicolas Sarkozy, 30 avril 2008, allocution lors de la remise du brin de muguet (Palais de l'Élysée).

23. Macron, 10 décembre 2018, allocution télévisée.

24. Macron, 25 avril 2019, conférence de presse.

25. Sarkozy, 13 mai 2008, discours devant les salariés de Yoplait en Isère.

26. Ibid.

27. Sarkozy, 16 octobre 2009, entretien dans Le Figaro.

28. Macron, 13 janvier 2019, lettre aux Français.

29. Par exemple Sarkozy, 29 mai 2007, discours devant l'UMP ; 24 mars 2009, discours à SaintQuentin ; 25 janvier 2010, interview à TF1. Macron, 29 mars 2018, discours à AI for Humanity ; 13 janvier 2019, Lettre aux Français.

30. On notera que Macron en cherchant à se démarquer de la formulation sarkozyste initiale en arrive à un énoncé contestable historiquement : depuis 150 ans, il semble que les sociétés modernes aient vu le temps de travail annuel, mensuel, hebdomadaire ou quotidien diminuer pour les travailleurs (congés payés, durée légale de travail, retraite, congé de maladie) en même temps que les salaires, le pouvoir d'achat et le niveau de vie augmentaient. Sur un temps long (XIX ${ }^{\mathrm{e}}, \mathrm{XX}^{\mathrm{e}}, \mathrm{XXI}^{\mathrm{e}}$ siècle), il semble donc bien, à strictement reprendre Macron, que l'on ait pu «travailler moins et gagner plus " grâce aux gains de productivité, à la mécanisation, au numérique. Mais l'énoncé de Macron ne doit pas être analysé au regard de la réalité historique : sa pertinence doit être évaluée par rapport au positionnement idéologique qu'il partage avec Sarkozy et que nous venons de décrire.

31. Voir par exemple N. Roussellier, La Force de gouverner. Le pouvoir exécutif en France, XIX ${ }^{e}$ $X_{X I}^{e}$ siècles, Gallimard, 2015. L'auteur rappelle les gènes et la double naissance de la Constitution ; le texte initial de 1958 puis la réforme de 1962. En apparence plus technique, l'inversion du calendrier entre les législatives et la présidentielle, voté en 2001, joue aussi un rôle important.

32. D'un point de vue philosophique comme sociologique, la montée en puissance de l'individualisme dans les sociétés contemporaines a été souvent décrite, au-delà des conséquences politiques sur la personnalisation du pouvoir. On lira, entre autres exemples, Jacques Guigou, La cité des ego, L'harmattan, 2008 (1987) et on se reportera aux ouvrages de Régis Debray qui décrivent cette société contemporaine du « tout-à-l'ego ».

33. Bernard Manin, Principes du gouvernement représentatif, Flammarion, 2012.

34. En l'occurrence, l'idée socialiste.

35. Patrick Charaudeau, Entre populisme et peopolisme. Comment Sarkozy a gagné, Paris, Vuibert, 2008.

36. . François Jost et Denis Muzet, Le Téléprésident, La Tour-d'Aigues, L'Aube, 2008 ; Cyril Lemieux, Un président élu par les médias? Paris, Presses des Mines, 2010 ; Éric Maigret, L’Hyperprésident, Paris, Armand Colin, 2008. De manière significative, dix ans après Sarkozy, les accointances de 
Macron avec les grands groupes de presse furent pointées par ses concurrents durant la campagne 2017 et plus encore durant le lancement de En Marche en 2016.

37. Cf. par exemple l'interview de Nicolas Sarkozy, le 22 aout 2007 dans Sud-Ouest.

38. Macron, 23 novembre 2017, discours aux Maires de France.

39. Macron, 7 mai 2017, discours de la victoire.

40. Bernard Manin, Principes du gouvernement représentatif, Flammarion, 2012.

41. Macron, 20 décembre 2018, contribution de M. Le Président de la République sur la pétition en ligne : https://www.change.org/p/pour-une-baisse-des-prix-à-la-pompe-essence-diesel/.

42. Macron, tweet du 14 avril 2019, 10h41. A l'intérieur du tweet, la bande annonce décline l'idée toujours sur le mode personnel en concluant: «Lundi 15 avril, à 20h, je vous réponds.»: Le linguiste aura remarqué l'usage performatif du présent de l'indicatif en guise de futur (« réponds »). Le lendemain, ce discours ne pourra finalement pas être tenu à cause de l'incendie des Notre-Dame de Paris.

43. Ainsi, par exemple, les garants du Grand débat avertissait: "L'implication personnelle du président de la République, son "hypermédiatisation", ont pu nourrir le doute sur la nature et l'objectif du grand débat national et ont en tout cas polarisé l'attention sur le chef de l'État en occultant pour le grand public la richesse de la parole publique qui en était le véritable enjeu. » (Nadia Bellaoui, discours du collège des garants du Grand débat national, 8 avril 2019, Grand palais de Paris).

44. F. Hollande, 31 mars 2019, interview sur France 2.

\section{RÉSUMÉS}

La logométrie et l'Intelligence artificielle (deep learning) appliquées aux textes politiques permettent de repérer dans le discours d'Emmanuel Macron les emprunts linguistiques qu'il contracte auprès de ses prédécesseurs à l'Elysée (de Gaulle, Pompidou, Giscard, Mitterrand, Chirac, Sarkozy et Hollande). Les emprunts les plus importants, lexicaux autour de la valeur travail et énonciatifs autour de l'exhibition du « je » et du « je veux », concernent statistiquement Nicolas Sarkozy.

Logometry and Artificial Intelligence (deep learning) applied to political texts make it possible to identify in Emmanuel Macron's speeches the linguistic borrowings he contracted from his predecessors at the Palais de l'Elysée (de Gaulle, Pompidou, Giscard, Mitterrand, Chirac, Sarkozy and Hollande). The most important borrowings, which are around the work value and enunciative around the exhibition of "I" and "I want", are statistically related to Nicolas Sarkozy.

\section{INDEX}

Mots-clés : discours présidentiel, discours politique, Intelligence artificielle, logométrie, Macron, Sarkozy

Keywords : presidential speech, political discourse, Artificial intelligence, deep learning, logometry, Macron 


\section{AUTEURS}

\section{DAMON MAYAFFRE}

Université Côte d'Azur, CNRS, BCL, France - damon.mayaffre@univ-cotedazur.fr MAGALI GUARESI

Université Libre de Bruxelles (ReSIC) - magali.guaresi@gmail.com

\section{LAURENT VANNI}

Université Côte d'Azur, CNRS, BCL, France - laurent.vanni@univ.cotedazur.fr 